\title{
Association of vitamin $D$ receptor gene polymorphisms with diabetic dyslipidemia in the elderly male population in North China
}

This article was published in the following Dove Press journal:

Clinical Interventions in Aging

10 October 2017

Number of times this article has been viewed

\author{
Zheng $\mathrm{Xia}^{\mathrm{l}, *}$ \\ Yazhuo $\mathrm{Hu}^{1, *}$ \\ Zhitao Han' \\ Ya Gao' \\ Jie $\mathrm{Bai}^{2}$ \\ Yao $\mathrm{He}^{\mathrm{I}}$ \\ Hua Zhao ${ }^{3}$ \\ Honghong Zhang' \\ 'Institute of Geriatrics, Beijing Key \\ Laboratory of Normal Aging and \\ Geriatrics, ${ }^{2}$ Cinical Laboratory in \\ South Building, Chinese PLA General \\ Hospital \& Chinese PLA Medical \\ Academy, Beijing, China; ${ }^{3}$ Zhantansi \\ Outpatient Department, Chinese PLA \\ 309 Hospital, Beijing, China
}

*These authors contributed equally to this work

\begin{abstract}
Background: The prevalence of dyslipidemia is rising alarmingly in elderly Han Chinese male patients with type 2 diabetes mellitus (T2DM). The genetic factors that contribute to the development of diabetic dyslipidemia remain incompletely identified. This study was conducted to assess the association between vitamin D receptor (VDR) polymorphisms and development of dyslipidemia in the Han elderly male population with T2DM in North China.
\end{abstract}

Methods: A total of 242 T2DM patients with dyslipidemia (DH group, n=108) or without dyslipidemia (DO group, $\mathrm{n}=134$ ) and 100 controls were genotyped for ApaI, TaqI and FokI single nucleotide polymorphisms (SNPs) of the VDR gene using polymerase chain reactionrestriction fragment length polymorphism and sequencing. The frequency and distribution of the SNPs were compared between cases and controls.

Results: The distribution of genotypes of VDR-FokI was significantly different between the control and DM group ( $P=0.033)$, as well as between the control and DH subgroup $(P=0.011)$ but not DO subgroup $(P=0.111)$. The frequency of $\mathrm{C}$ allele and $\mathrm{CC}$ genotype of FokI was significantly higher in the $\mathrm{DH}$ patients than in the controls ( $P=0.015$ and $P=0.003$, respectively). Logistic regression analysis in a dominant model homozygous for the $\mathrm{C}$ allele of the FokI SNP showed that CC genotype was associated with DH patients ( $\mathrm{OR}=1.797,95 \%$ CI: 1.077-2.999, $P=0.025$ ). Significant associations of the ApaI and TaqI SNPs with either DO or DH subjects were not observed.

Conclusion: These findings suggest that CC genotype of VDR-FokI is a risk factor for T2DM patients with dyslipidemia in elderly males in North China.

Keywords: vitamin D receptor, VDR, diabetes, dyslipidemia, polymorphism, elderly

\section{Introduction}

Type II diabetes mellitus (T2DM) is a multifactorial disease caused by both nongenetic and genetic factors. Nongenetic factors including environmental factors, lifestyle and medical conditions play an important role in the development of T2DM. ${ }^{1}$ For example, the majority of individuals with T2DM are either overweight or obese, and people who have hypertension and high triglyceride (TG) or cholesterol levels are more likely to develop T2DM. ${ }^{2}$ Inherited factors are also known to be important; however, up to now the genes predisposing patients to T2DM remain poorly defined. The major difficulties in clearly identifying the genes involved lie in the fact that the variations in the candidate genes are usually subtle and the contribution of each of these variations to the risk of developing diabetes depends on its interaction with other nongenetic and genetic factors. Thus, despite significant recent progress in genomic and statistical methodologies, the genetic dissection of human T2DM remains a challenge. 
Dyslipidemia, characterized by high levels of TG and very-low-density lipoprotein (VLDL) and low levels of highdensity lipoprotein (HDL) cholesterol in plasma, is one of the major risk factors for cardiovascular disease, the main cause of death in patients with diabetes mellitus. The lipid changes associated with diabetes mellitus are attributed to increased free fatty acid flux secondary to insulin resistance. On the other hand, the prevalence of dyslipidemia in those with diabetes mellitus was significantly higher than in the nondiabetic individuals, suggesting a risk linkage and adverse interaction between the two conditions.

One of the candidate genetic factors that may link the two conditions is the gene encoding vitamin D receptor (VDR). Vitamin D is essential for control of bone and calcium homeostasis, thus affecting calcium-regulated responses including lipid metabolism and immune response. ${ }^{3}$ Furthermore, it has been shown in the experimental animal models that vitamin D is necessary for the maintenance of glucose and normal insulin release, both of which are defective in T2DM. ${ }^{4,5}$ The active form of vitamin D is 1 , 25-dihydroxyvitamin D. Its level in cells and circulation is tightly regulated by VDR. ${ }^{6}$

The VDR gene is located on chromosome 12 cen-q12 and contains 11 exons. In recent years, several single nucleotide polymorphisms (SNPs) of the VDR gene have been reported to be associated with susceptibility to many diseases including diabetes. ${ }^{7}$ The most frequently reported VDR SNPs were VDR-FokI, VDR-BsmI, VDR-TaqI and VDR-ApaI that were initially identified by different restriction enzymes. Of these SNPs, the BsmI and ApaI polymorphisms resulted from the substitutions in intron 8, whereas Taq1 resulted from a substitution of cytosine (C) with thymine (T) in exon 9. All of these three SNPs are located near the 3'-3'-Untranslated Region ( $3^{\prime}$-UTR) region and are thought to alter the stability of the VDR mRNA. ${ }^{8}$ The ForkI restriction enzyme identifies a polymorphic site in exon 2 . More specifically, a $\mathrm{T}$ to $\mathrm{C}$ variation is found in the start codon of the VDR gene which can no longer be cut by ForkI. The change of ATG (FokI T) to ACG (FokI C) results in a VDR protein that is three amino acids shorter. ${ }^{9}$ Compared with the full-length VDR, this shorter form may have altered biological activity. ${ }^{10}$

VDR SNPs vary markedly in different ethnic groups and gender. ${ }^{11}$ For instance, GG is the primary VDR-ApaI genotype in Asian populations but not in European and African populations. For VDR-FokI, CT is the primary genotype in the Chinese Beijing population (54\%), while such genotype accounts for only $33 \%$ and $31 \%$ of the Japanese Tokyo population and African population, respectively.
The gender-specific effects of VDR SNPs on biological function and diseases were also observed. An association of VDR SNPs with bone mineral density and skeletal growth was only detected in girls but not in boys. ${ }^{12}$ In addition, gender also appears to influence the linkage of VDR SNPs with the phenotype of some immune-mediated diseases, including Type 1 diabetes mellitus. ${ }^{13}$ Given these observations, association between VDR SNPS and T2DM would benefit from examination of additional populations in the context of gender.

As lifestyle has been changing in the recent years in China, the prevalence of T2DM, especially in males, is notably increasing, and it is associated with an increased incidence of obesity and dyslipidemia in the population. ${ }^{14}$ Assessment of genetic risk factors for concurrence of T2DM and dyslipidemia in this population, however, is lacking. Promoted by this fact and above-mentioned findings that VDR may play a role in the etiology and pathogenesis of T2DM, we conducted a case-controlled study to evaluate the distribution and association of three VDR SNPs, including ApaI, Taq1 and FokI, in elderly male T2DM patients with or without dyslipidemia in the Han population of Northern China.

\section{Methods}

\section{Study design and subjects}

A total of 242 male T2DM (DM) patients (age $\geq 60$ years) and 100 age- and gender-matched healthy controls (ND) of Han nationality were enrolled in this study. Depending on their lipid profile in plasma, the 242 patients were further grouped into no dyslipidemia group (designated as DO, n=108) and with dyslipidemia group (designated as $\mathrm{DH}, \mathrm{n}=134$ ). All the enrolled individuals are permanent residents of Beijing. Informed consent was obtained from each subject prior to the study. The study was approved by the ethical review committee of the Chinese PLA General Hospital. Peripheral blood samples $(5 \mathrm{~mL})$ were collected from all the participants in EDTA collection tubes.

\section{Inclusion and exclusion criteria}

Inclusion criteria were as follows: 1) diagnosis of T2DM was based on the WHO diagnostic criteria for diabetes mellitus (1999), and 2) dyslipidemia was assessed based on the 2007 standard of "Chinese Adult Dyslipidemia Prevention Guide," which includes total cholesterol (TC) $\geq 5.18 \mathrm{mmol} / \mathrm{L}$ (200 mg/dL); low-density lipoprotein cholesterol (LDL-C) $\geq 3.37 \mathrm{mmol} / \mathrm{L}(130 \mathrm{mg} / \mathrm{dL})$; HDL cholesterol (HDL-C) $<1.04 \mathrm{mmol} / \mathrm{L}(40 \mathrm{mg} / \mathrm{dL})$ and 
$\mathrm{TG} \geq 1.7 \mathrm{mmol} / \mathrm{L}(150 \mathrm{mg} / \mathrm{dL})$. Exclusion criteria were as follows: 1) individuals from non-Han ethnic groups, healthy individuals with blood relationship with the T2DM subjects or with family history of diabetes, individuals with type 1 diabetes or secondary diabetes and individuals with familial hyperlipidemia; 2) individuals with thyroid disease, hyperuricemia and other metabolic diseases; 3 ) individuals with cancer, poisoning, liver disease, kidney disease, pancreatic disease, autoimmune diseases, infectious diseases, stress, skin diseases, major surgery or with serious mental illness; 4) individuals with severe cardiovascular diseases such as cerebrovascular accident, myocardial infarction, heart failure, atrioventricular block, sick sinus syndrome, aneurysm or artery dissection and arteriosclerosis obliterans; and 5) individuals taking glucocorticoid steroid hormone drugs. Data for exclusion criteria were derived from clinical medical records and questionnaire.

\section{Clinical laboratory analysis}

General information including history of dyslipidemia and diabetes, duration of disease and lifestyle of smoking and drinking (recorded as: $0=$ never, $1=$ now and $2=$ past) was collected from medical record or questionnaire. Body mass index (BMI) was calculated by the formula weight $(\mathrm{kg}) /$ height $^{2}\left(\mathrm{~m}^{2}\right)$. Systolic blood pressure and diastolic blood pressure were measured after rest for at least 15 minutes. Subjects from all groups were subjected to 8-10 hours fasting before the blood sample was collected for determination of glucose (FBG), lipid profile, urea nitrogen, creatinine, uric acid and alkaline phosphatase.

\section{Genotyping}

Total DNA was extracted from whole blood using DNA extraction kit (TIANGEN Company, Beijing, China). Genotyping for VDR SNPs including VDR-ApaI (rs7975232; T/G), VDR-TaqI (rs731236; C/T) and VDR-FokI (rs2228570; C/T) was performed using polymerase chain reaction-restriction fragment length polymorphism analysis followed by sequencing the representative samples in Shenggong Bioengineering Company (Shanghai, China) with primers and conditions described previously. ${ }^{15}$

\section{Statistical analysis}

SPSS version 20.0 for windows was used for statistical analysis. Hardy-Weinberg equation $\chi^{2}=\Sigma(o b-e x) 2 / e x$, where ob stands for observed data and ex stands for expected data, was used to determine whether the observed genotype or allele frequencies of VDR SNPs in the current study differ from those of the population predicted by the equation. Chi-squared test was used to compare cases and controls with qualitative data. Quantitative data were presented as mean \pm standard deviation and analyzed using independent samples $t$-test, ANOVA or Student-Newman-Keuls test. Statistically significant difference was defined as $P<0.05$. Linkage disequilibrium (LD) was analyzed with Haploview software (version 4.2). Unconditional logistic regression is a regression model where the dependent variable is categorical, which was used to estimate associations between each VDR SNP and T2DM (DO and DH) at 95\% confidence intervals (CIs).

\section{Results}

Clinical characteristics of the three groups are summarized in Table 1. The average age of the patients in the DO and DH groups was $77.2 \pm 9.8$ and $75.7 \pm 9.9$ years with duration of T2DM for $12.4 \pm 7.8$ and $11.9 \pm 8.9$ years, respectively. Among these patients, the DH group was classified by significantly abnormal lipid profile with high levels of TC, TG, LDL-C, non-HDL-C and LDL-C/HDL-C ratio and low HDL-C compared with the DO group and normal control group (ND). It should be noted that despite significantly abnormal lipid profile, the DH group was very similar to the DO group in FBG and 2-hour postprandial blood glucose levels $(6.2 \pm 1.1$ vs $6.2 \pm 1.5$ and $10.6 \pm 2.1$ vs $10.3 \pm 1.9$, respectively), suggesting the occurrence of dyslipidemia in this group is not directly related to hyperglycemia and potential involvement of genetic factors. In addition, compared with the ND group, BMI in patients with T2DM was higher and this difference was significant when compared with the $\mathrm{DH}$ group $(P=0.019)$.

Allele frequency and genotype distribution of VDRApaI, -Taq1 and -FokI are depicted in Tables 2, 3 and 4, respectively. All of them were in Hardy-Weinberg equilibrium, indicating that they are representative for the population (data not shown). There were no significant differences between diabetic and control subjects in the distribution of ApaI and Taq1 allelic SNPs and genotypes (Tables 2 and 3). However, the frequency of FokI allele $\mathrm{C}$ was significantly higher in the diabetic subjects compared with controls $(P=0.012$; Table 4). Unconditional logistic regression analysis showed the FokI allele $\mathrm{C}$ was significantly associated with T2DM (OR $=1.566,95 \%$ CI: $1.04-2.222, P=0.012)$. In addition, the distribution of genotypes of FokI was significantly different between ND and DM groups $(P=0.033)$. The CC genotype of FokI in a dominant model was significantly higher in the subjects with T2DM than in the controls $(P=0.010$; Table 5). Diabetic subjects with dyslipidemia 
Table I Clinical characteristics of case groups and controls

\begin{tabular}{|c|c|c|c|c|}
\hline Characteristics & ND $(\mathbf{N}=100)$ & $\mathrm{DO}(\mathrm{N}=134)$ & $\mathrm{DH}(\mathrm{N}=108)$ & $P$-value \\
\hline \multicolumn{5}{|l|}{ General characteristics } \\
\hline Age (years) & $75.8 \pm 11.2$ & $77.2 \pm 9.8$ & $75.7 \pm 9.9$ & 0.442 \\
\hline BMI $\left(\mathrm{kg} / \mathrm{m}^{2}\right)$ & $23.6 \pm 2.5$ & $24.1 \pm 2.5$ & $24.6 \pm 2.8^{*}$ & 0.019 \\
\hline $\mathrm{SBP}(\mathrm{mmHg})$ & $125.9 \pm 11.9$ & $127.8 \pm 12.6$ & $|28.8 \pm| \mid .8$ & 0.209 \\
\hline $\mathrm{DBP}(\mathrm{mmHg})$ & $71.3 \pm 8.9$ & $70.1 \pm 9.0$ & $71.5 \pm 7.7$ & 0.380 \\
\hline \multicolumn{5}{|l|}{ Diabetic characteristic } \\
\hline Duration of diabetes (years) & - & $12.4 \pm 7.8$ & $11.9 \pm 8.9$ & $0.70 \mathrm{I}$ \\
\hline $\mathrm{FBG}(\mathrm{mmol} / \mathrm{L})$ & $5.3 \pm 0.4$ & $6.2 \pm 1.1 *$ & $6.2 \pm 1.5^{*}$ & $<0.001$ \\
\hline $2 \mathrm{~h} \mathrm{PG} \mathrm{(mmol/L)}$ & $6.8 \pm 0.7$ & $10.6 \pm 2.1 *$ & $10.3 \pm 1.9 *$ & $<0.001$ \\
\hline \multicolumn{5}{|l|}{ Lipid profile } \\
\hline $\mathrm{TC}(\mathrm{mmol} / \mathrm{L})$ & $4.2 \pm 0.5$ & $4.1 \pm 0.6$ & $4.9 \pm 1.0^{*, \#}$ & $<0.00 \mathrm{I}$ \\
\hline TG (mmol/L) & $1.0 \pm 0.3$ & $1.0 \pm 0.3$ & $1.7 \pm 0.8^{*, \#}$ & $<0.001$ \\
\hline LDL-C (mmol/L) & $2.4 \pm 0.5$ & $2.4 \pm 0.6$ & $3.1 \pm 0.9 *, \#$ & $<0.001$ \\
\hline HDL-C (mmol/L) & $1.4 \pm 0.3$ & $1.4 \pm 0.3$ & $1.2 \pm 0.4^{*, \#}$ & $<0.00$ I \\
\hline Non-HDL-C (mmol/L) & $2.8 \pm 0.5$ & $2.7 \pm 0.6$ & $3.7 \pm 0.9 * \#$ & $<0.001$ \\
\hline LDL-C/HDL-C & $1.8 \pm 0.6$ & $1.8 \pm 0.5$ & $2.7 \pm 0.7^{* \# \#}$ & $<0.001$ \\
\hline \multicolumn{5}{|l|}{ Liver and kidney function } \\
\hline BUN (mmol/L) & $5.9 \pm 1.4$ & $6.1 \pm 1.4$ & $5.9 \pm 1.4$ & 0.175 \\
\hline $\mathrm{Cr}$ (umol/L) & $85.0 \pm 13.1$ & $84.3 \pm 15.0$ & $82.4 \pm 14.2$ & 0.400 \\
\hline UA (umol/L) & $334.0 \pm 64.8$ & $334.9 \pm 62.7$ & $349.4 \pm 72.9$ & 0.159 \\
\hline ALP (IU/L) & $17.4 \pm 8.4$ & $17.2 \pm 7.3$ & $18.3 \pm 8.6$ & 0.527 \\
\hline Smoking $(0 / 1 / 2)^{\mathrm{a}}$ & $72 / 16 / 12$ & $85 / 19 / 30$ & $61 / 23 / 24$ & 0.102 \\
\hline Drinking $(0 / 1 / 2)^{\mathrm{a}}$ & $70 / 25 / 5$ & $93 / 37 / 4$ & $77 / 27 / 4$ & 0.930 \\
\hline
\end{tabular}

Table 2 Comparison of VDR-Apal genotype and allele frequency between case groups and controls

\begin{tabular}{|c|c|c|c|c|c|c|}
\hline \multirow[t]{2}{*}{ Group } & \multirow[t]{2}{*}{$\mathbf{N}$} & \multicolumn{3}{|l|}{ Genotype } & \multicolumn{2}{|l|}{ Allele } \\
\hline & & TT, n (\%) & TG, n (\%) & GG, n (\%) & $\mathbf{T}, \mathbf{n}(\%)$ & G, n (\%) \\
\hline ND & 100 & $13(13)$ & $38(38)$ & $49(49)$ & $64(32)$ & $136(68)$ \\
\hline DM & 242 & $19(7.9)$ & $92(38)$ & $131(54.1)$ & $130(26.9)$ & $354(73.1)$ \\
\hline DO & 134 & $9(6.7)$ & $50(37.3)$ & $75(56)$ & $68(25.4)$ & $200(74.6)$ \\
\hline $\mathrm{DH}$ & 108 & $10(9.3)$ & $42(38.9)$ & $56(51.9)$ & $62(28.7)$ & I54 (7I.3) \\
\hline ND vs DM & & & & $\chi^{2}=2.359 P=0.307$ & & $\chi^{2}=1.84 \mid P=0.175$ \\
\hline $\mathrm{ND}$ vs $\mathrm{DO}$ vs $\mathrm{DH}$ & & & & $\chi^{2}=3.004 P=0.557$ & & $\chi^{2}=2.494 P=0.287$ \\
\hline
\end{tabular}

Abbreviations: ND, healthy controls; DM, diabetes mellitus; DO, type 2 diabetes mellitus patients without dyslipidemia; DH, type 2 diabetes mellitus patients with dyslipidemia; VDR, vitamin D receptor.

Table 3 Comparison of VDR-Taql genotype and allele frequency between case groups and controls

\begin{tabular}{|c|c|c|c|c|c|c|}
\hline \multirow[t]{2}{*}{ Group } & \multirow[t]{2}{*}{$\mathbf{N}$} & \multicolumn{3}{|l|}{ Genotype } & \multicolumn{2}{|l|}{ Allele } \\
\hline & & TT, n (\%) & TC, n (\%) & CC, n (\%) & $\mathrm{T}, \mathrm{n}(\%)$ & C, n (\%) \\
\hline ND & 100 & $86(86)$ & $14(14)$ & $0(0)$ & $186(93)$ & $14(7)$ \\
\hline DM & 242 & $224(92.6)$ & I8 (7.4) & $0(0)$ & $466(96.3)$ & $18(3.7)$ \\
\hline DO & 134 & $127(94.8)$ & $7(5.2)$ & $0(0)$ & $26 I(97.4)$ & $7(2.6)$ \\
\hline $\mathrm{DH}$ & 108 & 97 (89.8) & II (I0.2) & $0(0)$ & 205 (94.9) & II (5.I) \\
\hline ND vs DM & & & & $\chi^{2}=3.593 P=0.058$ & & $\chi^{2}=3.416 P=0.065$ \\
\hline$N D$ vs $D O$ vs $D H$ & & & & $\chi^{2}=5.328 P=0.070$ & & $\chi^{2}=5.067 P=0.079$ \\
\hline
\end{tabular}

Abbreviations: ND, healthy controls; DM, diabetes mellitus; DO, type 2 diabetes mellitus patients without dyslipidemia; DH, type 2 diabetes mellitus patients with dyslipidemia; VDR, vitamin D receptor. 
Table 4 Comparison of VDR-Fokl genotype and allele frequency between case groups and controls

\begin{tabular}{|c|c|c|c|c|c|c|}
\hline \multirow[t]{2}{*}{ Group } & \multirow[t]{2}{*}{$\mathbf{N}$} & \multicolumn{3}{|l|}{ Genotype } & \multicolumn{2}{|l|}{ Allele } \\
\hline & & CC, n (\%) & CT, n (\%) & TT, n (\%) & C, n (\%) & T, n (\%) \\
\hline ND & 100 & $38(38)$ & $50(50)$ & $12(12)$ & $126(63)$ & $74(37)$ \\
\hline DM & 242 & $129(53.3)$ & $94(38.8)$ & $19(7.9)$ & $352(72.7)$ & $132(27.3)$ \\
\hline DO & 134 & $66(49.3)$ & $60(44.8)$ & $8(6)$ & $192(7 \mid .6)$ & $76(28.4)$ \\
\hline $\mathrm{DH}$ & 108 & $63(58.3)$ & $34(31.5)$ & $11(10.2)$ & $160(74.1)$ & $56(25.9)$ \\
\hline ND vs DM* & & & & $\chi^{2}=6.830 P=0.033$ & & $\chi^{2}=6.362 P=0.012$ \\
\hline ND vs $D O$ vs $\mathrm{DH}^{*}$ & & & & $\chi^{2}=\mid 1.522 P=0.021$ & & $\chi^{2}=6.699 P=0.035$ \\
\hline ND vs DO & & & & $\chi^{2}=4.400 P=0.111$ & & $\chi^{2}=3.927 P=0.048$ \\
\hline $\mathrm{ND}$ vs $\mathrm{DH}^{*}$ & & & & $\chi^{2}=8.985 P=0.011$ & & $\chi^{2}=5.928 P=0.015$ \\
\hline DO vs DH & & & & $\chi^{2}=4.999 P=0.082$ & & $\chi^{2}=0.357 P=0.550$ \\
\hline
\end{tabular}

Note: *Indicates significant difference.

Abbreviations: ND, healthy controls; DM, diabetes mellitus; DO, type 2 diabetes mellitus patients without dyslipidemia; $\mathrm{DH}$, type 2 diabetes mellitus patients with dyslipidemia; VDR, vitamin D receptor.

(DH group) appeared to be the major contributors to the previously mentioned differences. The frequency of FokI allele $\mathrm{C}$ and $\mathrm{CC}$ genotype was higher in $\mathrm{DH}$ subgroup than DO subgroup of the diabetic subjects $(74.1 \%$ vs $71.6 \%$ and $58.3 \%$ vs $49.3 \%$, respectively), which differed significantly from controls, whereas these differences were less significant when comparing DO group with controls. Indeed, logistic regression analysis revealed that the CC genotype of FokI was only statistically associated with $\mathrm{DH}$ group $(\mathrm{OR}=1.984$, 95\% CI: $1.080-3.646, P=0.027)$ but not with DO group (OR $=1.717,95 \%$ CI: $0.961-3.070, P=0.068)$.

In the present study, LD among the three VDR SNPs was evaluated using Haploview software. As shown in Figure 1, no strong LD was observed. In addition, there was no statistically significant association of the genotypes of three VDR SNPs with any of the individual clinical characteristics listed in Table 1, except an association of FokI TT genotype with increased TG level $(P=0.008)$. This association, however, is less reliable due to a limited number of FokI TT genotypes in the study (data not shown).

\section{Discussion}

It is well known that in the pathogenesis of diabetes, hyperglycemia and dyslipidemia are the most important acquired factors that act additively to accelerate the disease progression. The major pathogenic basis for hyperglycemia is impaired pancreatic $\beta$-cell function and decreased insulin sensitivity, so-called insulin resistance which often becomes severe with increasing age and weight and is accompanied by various metabolic disorders, the most common being dyslipidemia. Dyslipidemia is not only the major pathogenic basis for a variety of vascular complications of diabetes, but is also known to contribute to the decreased insulin sensitivity in diabetes. To better understand the development of T2DM, it is important to identify the genetic risk factors for the two combined conditions.

Our study showed that VDR-FokI polymorphism C was dominant (63\%) in elderly male healthy subjects of Han nationality, and the distribution of VDR-FokI genotype in this group was $38 \%$ with CC, $50 \%$ with $\mathrm{CT}$ and $12 \%$ with TT. This result is consistent with another investigation

Table 5 Comparison of VDR-Fokl genotype between cases and controls in dominant or recessive model

\begin{tabular}{|c|c|c|c|c|c|}
\hline \multirow[t]{2}{*}{ Group } & \multirow[t]{2}{*}{$\mathbf{N}$} & \multicolumn{2}{|l|}{ Dominant } & \multicolumn{2}{|l|}{ Recessive } \\
\hline & & CC, n (\%) & CT + TT, n (\%) & $\mathrm{CC}+\mathrm{CT}, \mathrm{n}(\%)$ & TT, n (\%) \\
\hline ND & 100 & $38(38)$ & $62(62)$ & $88(88)$ & $12(12)$ \\
\hline DM & 242 & $129(53.3)$ & II3 (46.7) & $223(92.1)$ & $19(7.9)$ \\
\hline DO & 134 & $66(49.3)$ & $68(50.7)$ & $126(94)$ & $8(6)$ \\
\hline $\mathrm{DH}$ & 108 & $63(58.3)$ & $45(41.7)$ & 97 (89.8) & II (I0.2) \\
\hline ND vs DM* & & & $\chi^{2}=6.634 P=0.010$ & & $\chi^{2}=1.478 P=0.224$ \\
\hline ND vs $D O$ vs $D^{*}$ & & & $\chi^{2}=8.607 P=0.014$ & & $\chi^{2}=2.767 P=0.251$ \\
\hline ND vs DO & & & $\chi^{2}=2.937 P=0.087$ & & \\
\hline $\mathrm{ND}$ vs $\mathrm{DH}^{*}$ & & & $\chi^{2}=8.594 P=0.003$ & & \\
\hline DO vs DH & & & $\chi^{2}=1.981 \quad P=0.159$ & & \\
\hline
\end{tabular}

Note: *Indicates significant difference.

Abbreviations: ND, healthy controls; DM, diabetes mellitus; DO, type 2 diabetes mellitus patients without dyslipidemia; $\mathrm{DH}$, type 2 diabetes mellitus patients with dyslipidemia; VDR, vitamin D receptor. 


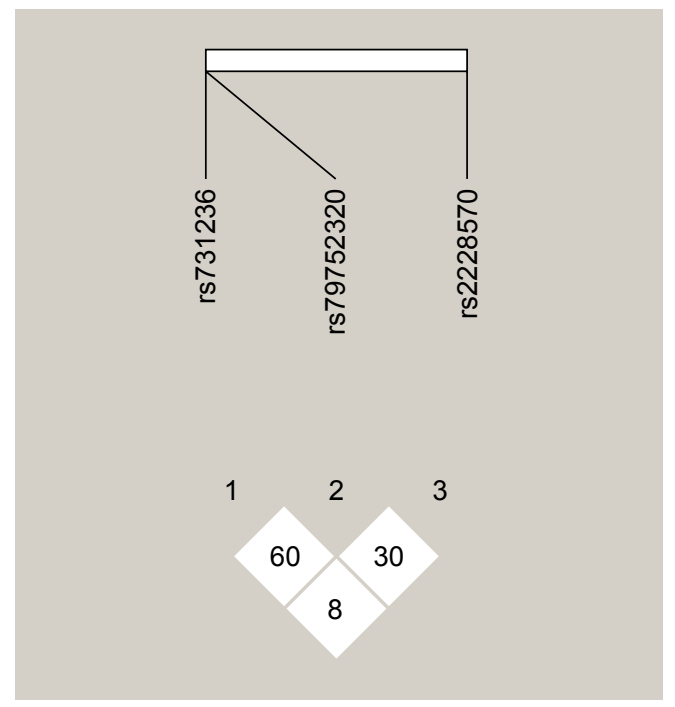

Figure I Pairwise linkage disequilibrium analysis of the three VDR gene SNPs: plot shows pairwise correlation of the SNPs (Taql, Apal and Fokl) studied.

Abbreviations: VDR, vitamin D receptor; SNPs, single nucleotide polymorphisms.

reported by Xin et $\mathrm{al}^{16}$ in which male subjects of the Han nationality in Beijing area were investigated for association of VDR-FokI with bone density, indicating that the subjects enrolled in the current study well represented the group. Compared with the controls, the frequency of FokI C allele and CC genotype in T2DM subjects was significantly higher, suggesting an association between VDR-FokI allele $\mathrm{C}$ and CC genotype and occurrence of T2DM in elderly males of the Han nationality. However, notably, although both DO and DH groups were found to have an increased FokI CC genotype compared with controls, the statistical significance was only associated with the diabetic subjects who also have dyslipidemia. Thus, our results suggest that FokI C, especially the CC genotype, is a risk factor for T2DM with dyslipidemia in elderly males of the Han nationality, whereas this association is less clear in the diabetic patients without dyslipidemia.

In contrast to the FokI polymorphism, VDR-ApaI and VDR-Taq1 SNPs are synonymous and have been proposed to affect the stability of mRNA of VDR. The frequencies of ApaI and Taq1 allele and genotype determined in the healthy controls of the current study were consistent with those reported by others as well as the National Institutes of Health's database for SNPs (http://www.ncbi.nlm.nih.gov/ projects/SNP/), indicating they represent the population. Compared with controls, the allele and genotype frequencies of VDR-ApaI and Taq1 SNPs in DM subjects were not significantly different.
The molecular mechanism underlining the association of VDR-FokI allele $\mathrm{C}$ with T2DM remains to be defined. Among the VDR SNPs investigated, VDR-FokI is the only polymorphism that is located in the coding sequence of the gene, thus resulting in altered VDR protein products. More specifically, the SNP of FokI consists of a $\mathrm{T}$ to $\mathrm{C}$ variation at translation initiation codon in exon 2 within the $5^{\prime}$ end of the gene. The change of ATG to ACG results in a three amino acids smaller protein with altered biological activity. However, the reason for the difference in activity of the two proteins, for example, whether they bind to 1,25 -hydroxyvitamin $\mathrm{D}$ differently is not clearly determined.

It has been found that low level of vitamin D is associated with decreased insulin sensitivity and increased insulin resistance. ${ }^{17,18}$ Vitamin D is also known to regulate lipid metabolism in adipocytes and fatty acid oxidation. It inhibits lipid synthesis and promotes adipopexis in adipocytes. ${ }^{19}$ Accordingly, it was noted in healthy Polish males that the allele $\mathrm{C}$ is associated with significantly decreased insulin sensitivity and HDL-C level. ${ }^{20}$ Similarly, a study in Caribbean population showed that vitamin D deficiency, was particularly prevalent in people with T2DM and those subjects carrying the $\mathrm{CC}$ genotype were more susceptible to vitamin D deficiency, which is closely related to increased level of TG. ${ }^{21}$ Alternatively, in addition to possible predeposition to insulin resistance and lipid metabolic disorder, VDR-FokI polymorphism has also been suggested to play a role in regulation of inflammation and immune function. ${ }^{22}$ Several groups have reported that VDR-FokI polymorphism is associated with cancers and autoimmune diseases, although the mechanisms remain to be determined. ${ }^{23}$ Considering autoimmune response and chronic inflammation may be involved in the destruction of cells in T2DM, it is possible that VDR-FokI polymorphism exerts its effect on T2DM with dyslipidemia via dysregulation of the immune system and inflammation. Future study in larger T2DM population with confined groups and detailed information regarding VDR level and activity, insulin secretion and sensitivity and autoimmunity to cells will help to dissect the underlying mechanism.

\section{Conclusion}

It is well known that in pathogenesis of diabetes, hyperglycemia and dyslipidemia are the most important acquired factors that act additively to accelerate the disease progression. Our study suggests that VDR-FokI genotype CC is a risk factor for T2DM combined with dyslipidemia in elderly male Chinese of the Han nationality. 


\section{Acknowledgments}

We are very grateful to all the participants of the study. This study was supported by the Special Fund for Innovation of Military Medical (13CXZ029). We acknowledge the primary care physicians and nurses for their cooperation in recruiting and collecting the data of the subjects.

\section{Disclosure}

The authors report no conflicts of interest in this work.

\section{References}

1. Lusis AJ, Attie AD, Reue K. Metabolic syndrome: from epidemiology to systems biology. Nat Rev Genet. 2008;9(11):819-830.

2. Lopez-Candales A, Hernández Burgos PM, Hernandez-Suarez DF, Harris D. Linking chronic inflammation with cardiovascular disease: from normal aging to the metabolic syndrome. J Nat Sci. 2017;3(4): pii:e341.

3. Pludowski P, Holick MF, Pilz S, et al. Vitamin D effects on musculoskeletal health, immunity, autoimmunity, cardiovascular disease, cancer, fertility, pregnancy, dementia and mortality - a review of recent evidence. Autoimmun Rev. 2013;12(10):976-989.

4. Cade C, Norman AW. Vitamin D3 improves impaired glucose tolerance and insulin secretion in the vitamin D-deficient rat in vivo. Endocrinology. 1986;119(1):84-90.

5. Yu F, Cui LL, Li X, et al. The genetic polymorphisms in vitamin D receptor and the risk of type 2 diabetes mellitus: an updated meta-analysis. Asia Pac J Clin Nutr. 2016;25(3):614-624.

6. Christakos S, Dhawan P, Verstuyf A, et al. Vitamin D: Metabolism, molecular mechanism of action, and pleiotropic effects. Physiol Rev. 2016;96(1): 365-408.

7. Jia J, Ding H, Yang K, et al. Vitamin D receptor genetic polymorphism is significantly associated with risk of type 2 diabetes mellitus in Chinese Han population. Arch Med Res. 2015;46(7):572-579.

8. Ingles SA, Haile RW, Henderson BE, et al. Strength of linkage disequilibrium between two vitamin D receptor markers in five ethnic groups: implications for association studies. Cancer Epidemiol Biomarkers Prev. 1997;6(2):93-98.

9. Saijo T, Ito M, Takeda E, et al. A unique mutation in the vitamin D receptor gene in three Japanese patients with vitamin D-dependent rickets type II: utility of single-strand conformation polymorphism analysis for heterozygous carrier detection. Am J Hum Genet. 1991;49(3):668-673.
10. Arai H, Miyamoto K, Taketani Y, et al. A vitamin D receptor gene polymorphism in the translation initiation codon: effect on protein activity and relation to bone mineral density in Japanese women. J Bone Miner Res. 1997;12(6):915-921.

11. Lins TC, Vieira RG, Grattapaglia D, et al. Population analysis of vitamin D receptor polymorphisms and the role of genetic ancestry in an admixed population. Genet Mol Biol. 2011;34(3):377-385.

12. Kitagawa I, Kitagawa Y, Kawase Y, et al. Advanced onset of menarche and higher bone mineral density depending on vitamin $\mathrm{D}$ receptor gene polymorphism. Eur J Endocrinol. 1998;139(5):522-527.

13. Györffy B, Vásárhelyi B, Krikovszky D, et al. Gender-specific association of vitamin $\mathrm{D}$ receptor polymorphism combinations with type 1 diabetes mellitus. Eur J Endocrinol. 2002;147(6):803-808.

14. Xu Y, Wang L, He J, et al. Prevalence and control of diabetes in Chinese adults. JAMA. 2013;310(9):948-959.

15. Maalmi H, Sassi FH, Berraies A, et al. Association of vitamin D receptor gene polymorphisms with susceptibility to asthma in Tunisian children: a case control study. Hum Immunol. 2013;74(2):234-240.

16. Han X, Zhan ZW, Zhang HH. Association between vitamin D receptor genotypes of FokI polymorphism and bone mineral density in male of the Han nationality in Beijing area. Zhongguo Zu zhi Gong cheng Yan jiu yu Lin chuang Kang fu. 2009;13(24):4763-4766.

17. Karnchanasorn R, Ou HY, Chiu KC. Plasma 25-hydroxyvitamin D levels are favorably associated with beta-cell function. Pancreas. 2012;41(6):863-868.

18. Pirgon $\mathrm{O}, \mathrm{Cekmez} F$, Bilgin $\mathrm{H}$, et al. Low 25 -hydroxyvitamin $\mathrm{D}$ level is associated with insulin sensitivity in obese adolescents with non-alcoholic fatty liver disease. Obes Res Clin Pract. 2013;7(4):e275-e283.

19. Sun X, Zemel MB. Role of uncoupling protein 2 (UCP2) expression and 1alpha, 25-dihydroxyvitamin D3 in modulating adipocyte apoptosis. FASEB J. 2004;18(12):1430-1432.

20. Filus A, Trzmiel A, Kuliczkowska-Plaksej J, et al. Relationship between vitamin D receptor BsmI and FokI polymorphisms and anthropometric and biochemical parameters describing metabolic syndrome. Aging Male. 2008;11(3):134-139.

21. Velayoudom-Cephise FL, Larifla L, Donnet JP, et al. Vitamin D deficiency, vitamin D receptor gene polymorphisms and cardiovascular risk factors in Caribbean patients with type 2 diabetes. Diabetes Metab. 2011;37(6):540-545.

22. Kamen DL, Tangpricha V. Vitamin D and molecular actions on the immune system: modulation of innate and autoimmunity. $J$ Mol $\operatorname{Med}(B e r l) .2010 ; 88(5): 441-450$.

23. Dankers W, Colin EM, van Hamburg JP, et al. Vitamin D in autoimmunity: molecular mechanisms and therapeutic potential. Front Immunol. 2017;20;7:697.
Clinical Interventions in Aging

\section{Publish your work in this journal}

Clinical Interventions in Aging is an international, peer-reviewed journal focusing on evidence-based reports on the value or lack thereof of treatments intended to prevent or delay the onset of maladaptive correlates of aging in human beings. This journal is indexed on PubMed Central, MedLine,

\section{Dovepress}

CAS, Scopus and the Elsevier Bibliographic databases. The manuscript management system is completely online and includes a very quick and fair peer-review system, which is all easy to use. Visit http://www.dovepress. com/testimonials.php to read real quotes from published authors. 